\title{
Clinical Development of Antiepileptic Drugs for Children
}

\author{
Elizabeth Garofalo
}

University of Michigan School of Medicine, Ann Arbor, Michigan 48103

\begin{abstract}
Summary: A clinical development plan specific to children is a necessary component of every development plan for a new antiepileptic drug (AED). In the last decade, considerable discussion has occurred in the medical and regulatory communities, resulting in specific pediatric drug development legislation. Ethical issues are a foremost consideration in the design and conduct of studies. The timing of clinical studies differs between adults and children. In general, studies in children will not be performed until efficacy and safety has been demonstrated in adults. Exceptions include development of AEDs for seizure types seen only in children. Formulation preparation and dosing selection are often more challenging in children.
\end{abstract}

Clinical trials including pharmacokinetic studies will be conducted in patients. A relatively small number of children, given subdivision into age groups and seizure types, are available for study. Clinical trials must be designed with children in mind, adjusting the length of the trials and the choice of controls. Efficacy extrapolation from adults may be considered for partial seizures in children, but not in infants. Seizure counts remain an appropriate efficacy endpoint; however, ascertainment in infants and younger children may require EEG monitoring. Safety specific to growing and developing children must be evaluated and long-term effects monitored. Key Words: Epilepsy, children, antiepileptic drugs, clinical development.

\section{INTRODUCTION}

Steady progress has been made in the area of drug development for children in the last decade, spurred by growing interest in both the United States and Europe, as well as legislation passed in the mid-1990s in the United States. In general, this progress has included more trials being performed, resulting in efficacy, safety, and dosing data generated from pediatric patients treated with new antiepileptic drugs (AEDs). Many challenges exist in completing a comprehensive development program in a timely fashion in children. ${ }^{1}$ Issues such as the timing of pediatric trials relative to trials in adults, the need for additional toxicology studies, and the development of one or more suitable formulations for children need to be addressed. There is clear recognition that when a medication is likely to be used in children it is imperative that appropriate studies be performed in this patient population. This principle has been articulated by regulatory authorities in Guidance ICH E 11, the general principles of which are summarized here (TABLE 1). This guidance characterizes the scope of the work and the special considerations unique to clinical development for chil-

Address correspondence and reprint requests to: Elizabeth Garofalo, MD, 1715 Newport Creek Drive, Ann Arbor, MI 48103. E-mail: Garofalo@umich.edu. dren in general. ${ }^{2}$ Although pediatric clinical development will vary for each new AED compound under study, some general principles can be elaborated.

\section{Regulatory background}

The regulatory environment for AED drug development has changed a great deal over the past decade, and some of the greatest changes have been seen in pediatric drug development. After initial legislation, the Best Pharmaceuticals for Children Act (BPCA) and the Pediatric Research Equity Act (PREA) were passed in 2002 and 2003, respectively., ${ }^{3,4}$ Similar legislation is under development in the European Union. PREA legislates that AEDs must be studied in children. PREA also defines the components necessary for a development program in children. BPCA offers an incentive to perform pediatric studies defined in a written request by granting the compound 6 months exclusivity. The BPCA will sunset in 2007.

\section{Ethical issues}

Although continued unmet medical need exists for the study of AEDs in children, ethical considerations dictate certain aspects of trial design and timing of trials in children relative to adults. Children must be protected as research participants. Studies in children must be designed with clearly defined and articulated risks and benefits. Standards are in place in order to evaluate the risks 
TABLE 1. General Principles (ICH E 11)

- Pediatric patients should be given medicines that have been properly evaluated for their use in the intended population

- Product development programs should include pediatric studies when pediatric use is expected

- Pediatric development should not delay adult studies nor adult availability

- Shared responsibility among companies, regulatory authorities, health professionals, and society as a whole

Source: U.S. Food and Drug Administration, Center for Drug Evaluation and Research, Center for Biologics Evaluation and Research (FDA-CDER-CBER), 2000. ${ }^{2}$

and benefits to the pediatric patients being studied, as well as to the population of children as a whole. ${ }^{5}$ These must then be included in the discussion with children and their families when informed consent is obtained-before children are entered into trials.

A workshop on AED drug trials in children was held in 1990 and called for pharmacokinetic (PK) studies to be initiated immediately after PK studies in adults, early in the clinical development process. ${ }^{6}$ It is highly questionable to expose pediatric patients to the risk and discomfort associated with PK studies prior to ascertaining proof of efficacy in adults. Many drugs introduced into initial PK studies in adults do not ultimately make it through the development process. If these drugs are indeed abandoned in later phases of development based on lack of efficacy, or unacceptable side effects, children enrolled in such early PK studies would be exposed to new chemical entities with no benefit to them, nor to society as a whole.

\section{Timing issues}

Pediatric studies of AEDs in seizure types seen in both adults and children should not, in general, be initiated with a new chemical entity prior to the establishment of the adult dose, serum concentration profile, and a commitment to take the compound forward to registration.

Adult PK and in vitro metabolism data are necessary for planning rational pediatric PK studies. Once it is clear that a given PK profile is associated with the desired therapeutic endpoint and an understanding of the safety of the compound has been determined in adults, then one can rationally and safely plan pediatric studies. A logical exception arises for seizure types not seen in adults, such as infantile spasms, in which case drug development may proceed from PK and early safety studies in adults to PK studies in children. At a U.S. National Institutes of Health consensus conference held in 1994, members called for pediatric PK data and pediatric dosing schedules to be made available at the time of approval (TABLE 2). ${ }^{7}$ The availability of an appropriate formulation for children will also factor into the start of pediatric studies.

\section{FORMULATION AND TOXICOLOGY}

Prior to initiation of trials, a single formulation (or possibly several different formulations) must be developed for children of different ages. This is a resourceintensive and time-consuming process. Initial development work for a commercial pediatric dosage formulation takes in general over 1 year to complete. Development teams plan on 1-1.5 years to complete manufacturing of small amounts of supplies for initial stability, initial clinical studies, and prototype refinement. Additional time will be required to complete the development process for commercial manufacturing. Further steps include transfer of the manufacturing process to commercial sites, manufacture of product for remaining clinical studies, and obtaining stability data for regulatory filings. An additional 2 years are needed to complete a stability program, in order to have an acceptable shelf life at the time of registration (12-18 months).

This work, which can be expected to take at least 4 years, must be completed in advance of a pediatric submission. In a resource-constrained environment, the work will often not begin until clear efficacy and safety data are available in adults. Furthermore, there are challenges of bioavailability, and palatability, along with the inherent issues of liquid formulations and the need for small milligram doses and small dosage form sizes.

TABLE 2. NIH Consensus Conference Recommendations

1. Antiepileptic drugs are needed for pediatric trials; trials for new antiepileptic drugs should include trials in children.

2. If a drug is shown to be effective for partial seizures in adults, this is sufficient to justify approval for use in partial seizures in children, provided that safety and pharmacokinetic considerations are appropriate.

3. Because antiepileptic drugs that are approved for use in adults are certain to be used in children,

pharmacokinetic data and dosing schedules for children should be provided to the professional community at the time of drug approval.

4. Putative antiepileptic drugs should be tested in immature animals as well as adult animals, to identify agents that may be age-specific. New models should be developed for specific age-related syndromes.

5. The development and standardization of new outcome measures of drug effects, especially in the areas of cognition and behavior, are recommended for the evaluation of new antiepileptic drugs in pediatric epilepsy.

6. Postmarketing surveillance for long-term development and cognitive and other safety issues needs to be developed for all new antiepileptic drugs.

7. Governmental agencies, such as the National Institute of Neurological Disorders and Stroke, are strongly urged to identify and support a system or systems to identify and track patients for antiepileptic drug development.

Source: Sheridan and Jacobs, $1996{ }^{7}$ 
These issues are highlighted and discussed in detail by the European Medicines Agency in a guideline publication from $2005 .^{8}$

Every clinical trial is supported by nonclinical toxicology studies in animals. Additional work in animals may be necessary to support a pediatric program, particularly if a safety signal is seen in the standard studies. This necessary work may also influence the onset of clinical trials in children.

\section{CLINICAL TRIALS}

\section{Dose selection}

Dose selection can be achieved by modeling PK characteristics associated with optimum efficacy and safety in adults, with efficacy extrapolation to children. This provides an efficient approach to minimizing the number of children who need to be studied. Studies in children are in patients, not in normal volunteers. They tend to be studies of fewer patients, using sparse blood sampling techniques, and thus are not as elegant as adult studies. Population PK sampling has been used successfully in AED development for children. ${ }^{9}$ Population PK studies are done in clinical settings and so may reflect the real world better than the studies done in traditional normal volunteers. These approaches will minimize the blood volumes that need to be obtained from each individual child, and, in general, avoid the need to do dose ranging studies.

\section{Patient selection}

Somewhat arbitrary age categories have been defined for the pediatric group: neonate ( $0-1$ month), infant (1 month-2 years), child (2-12 years), and adolescent (12-16 years). ${ }^{2}$ Both PK and efficacy trials should be proposed based on relevant information. Pharmacokinetic studies should be based on the application of knowledge of the ontogeny of the clearance pathways of the drug in different ages. Clinical trials in children should be based on the seizure type or epilepsy syndrome under study and should include children of appropriate ages.

Epidemiological data are available to help define the number of children with specific seizure types available for potential entry into a trial. The numbers vary by age, consistent with the onset of specific seizure types and epilepsy syndromes. Overall, 30,000 children can be estimated to be diagnosed with epilepsy annually in the United States. ${ }^{10}$ Of these 30,000 children, benign partial epilepsy accounts for 3000 children, or $10 \%$. In a population-based cohort study from Nova Scotia, $83 \%$ of children with new-onset partial seizures came under control with the first AED. ${ }^{11}$ Approximately 4000 children can be estimated to present with absence seizures and
1500 adolescents present with juvenile myoclonic epilepsy (JME) per year in the United States. ${ }^{10}$

These generalized epilepsy syndromes usually present in children of ages 6 years and older. Generalized tonicclonic seizures appear to be rare in infants. In one study of 69 infants less than 2 years of age with chronic seizures, none were found to have video-EEG confirmation of generalized tonic-clonic seizures. ${ }^{12}$ Statistics for complete response to therapy for absence epilepsy and JME are reported to range from $80 \%$ to $90 \% .^{13}$ Therefore, for both partial and generalized seizure disorders and epilepsies, only a small number of children will fail initial drug therapy and become medically refractory.

Partial seizures do occur in all pediatric age categories. Generalized epilepsy encompasses several seizure types. Studies of absence seizures would include school-age children, studies of JME would include adolescents, and studies of Lennox-Gastaut syndrome (with various seizures associated) would include infants through adults.

The neonatal patient represents a unique challenge for PK and clinical trials. The neonatal population is not homogeneous, and includes both preterm and term infants. Studies may therefore need to be done in several different patient strata, based on gestational age and weight. The neonatal patient is usually acutely ill, and seizures are often symptomatic and due to an underlying CNS or metabolic insult. ${ }^{11}$ Trials in this age group would center on acute intervention.

\section{Trial designs}

Trials for patients with refractory epilepsy-partial seizures. Randomized, adjunctive therapy trials are suitable for infants and children with refractory seizures. These studies have been performed and information included in labeling for at least three AEDs (Keppra; UCB, Brussels Belgium; Neurontin; Pfizer, New York, NY; Trileptal; Novartis, East Hanover, NJ) approved since 1994. ${ }^{14,15}$

Historically, in studies of adults, the baseline phase ranges from $8-12$ weeks and the treatment phases have been $8-12$ weeks in duration. Patients have been randomized to drug or placebo (see Schmidt in this issue). An analysis of data from several trials has shown that the baseline phase and treatment phase may be shortened and still allow for an acceptable determination of seizure rates and treatment effect. ${ }^{16}$ This is particularly relevant for children, allowing for a shorter overall trial and minimizing the amount of time before active treatment is available for each patient. The treatment phase needs to include time for therapy titration and attainment of steady-state drug concentrations, followed by sufficient quantification of seizure counts to justify chronic therapy.

In infants, a shorter baseline and treatment phase may be used, while still allowing for titration and evaluation at steady state. This allows for a shorter phase in which 
the patient may be exposed to placebo and a shorter time for evaluation of continuing seizures commensurate with the overall duration of the infant's illness. This shorter duration results in an increase in the number of patients enrolled in the trial who do not fulfill the requisite seizure frequency or who are entirely seizure free during the baseline phase. ${ }^{17}$ Consideration for replacement or other adjustment in sample-size determination needs to account for this eventuality.

Justification for the control group therapy is needed and varies with the question posed by the trial. Placebo and dose-controlled studies are acceptable alternatives for determination of efficacy. In studies in children, as in adult studies, seizure counts are designated as the primary outcome variable in determination of efficacy (see Schmidt, in this issue). Children and adolescents (2-16 years) can use diaries, much like adults. Accurately classifying seizures in pediatric patients or subjects can be more difficult with younger children than with older children and adolescents. ${ }^{18}$ Initial EEG diagnosis and seizure ascertainment may be necessary to accurately quantify seizures during the trial. Seizures in infants are difficult to classify and count clinically; accurate seizure classification and quantification can be ascertained by EEG. Video-EEG monitoring has been successfully used in clinical trials with infants. ${ }^{17}$

Trials for patients with refractory epilepsy-generalized seizures. Patients with Lennox-Gastaut syndrome and drop attacks have been successfully studied in randomized, placebo-controlled trials, with baseline phases ranging from 4 to 12 weeks and treatment phases from 11 to 16 weeks. Most trials have enrolled both children and adults in the same study. Outcome measures have included counts of drop attacks, EEG monitoring for seizures, and EEG changes. ${ }^{19,20}$ Medically refractory primary generalized tonic-clonic (PGTC) seizures have also been studied in at least one randomized, placebocontrolled trial with an 8 -week baseline phase and a 12-week treatment phase. ${ }^{21}$

Trials for patients with new-onset epilepsy. Ideally, trials in newly diagnosed patients would be performed as monotherapy. A prerandomization phase is necessary to ensure the diagnosis. The length of the treatment phase needs to be consistent with the likelihood of seizure recurrence, longer for partial seizures (months, years) and shorter for absence seizures (days, weeks). ${ }^{22-24}$ Ethical concerns have led to the choice of an active or dose-controlled as opposed to a placebo-controlled design. ${ }^{1,15,17}$

Trials of new-onset monotherapy for partial seizures need a long observation phase due to the infrequent occurrence of seizures. In one monotherapy trial in children with partial seizures, more than half of the patients enrolled had no seizures during the 5-day treatment phase (Trileptal, video-EEG evaluation). ${ }^{15}$ This elimi- nated the ability to detect a treatment effect. The long observation period needed limits the possibility of a placebo-controlled design. A dose-controlled design may be used. An exception to this has been in the case of benign epilepsy syndromes, such as rolandic epilepsy, for which a placebo-controlled trial may be considered. ${ }^{22}$

New-onset typical absence seizures have been studied in randomized, placebo-controlled studies of several weeks' duration and responder-enriched, placebo-controlled monotherapy designs. The treatment phase has been limited to a few weeks, for ethical reasons, and EEG monitoring has been used, with activation by hyperventilation in one case. ${ }^{23,24}$

Infantile spasms (IS) are a seizure type unique to infants. Many trials have been performed, but relatively few have been prospective, randomized, or controlled. Endpoints have varied between complete cessation of spasms, resolution of hypsarrythmia, determination of relapse rate, or other outcomes. Small numbers of patients have been included in studies, and choice of control therapy and treatment duration has varied. ${ }^{25}$

\section{Study sites}

An optimal study site includes an experienced principal investigator and study site personnel. Access to patients with the seizure type under study is critical. The small patient population necessitates potentially long enrollment duration or a large number of study investigator sites, often in multiple countries. ${ }^{26}$ It is much more efficient to have a smaller number of investigators enrolling a larger number of patients. Once an investigator has enrolled a single patient, substantial resources must be dedicated to the ongoing conduct of the trial at that site. Additional complexity is added by the need for videoEEG monitoring and the need for a blinded, central interpretation of EEG data. All of these issues increase the level of difficulty in conducting trials in children.

\section{SPECIAL SAFETY ISSUES IN CHILDREN}

Common adverse events as well as long-term safety may differ between adults and children. Therefore, collection of safety data and evaluation in the appropriate pediatric populations is critically important. The ability to collect meaningful data in terms of growth and development as well as behavior and cognitive function is complicated by the patient populations under study and the short duration of the efficacy trials. Longer follow-up may be obtained, but a lack of appropriate information about the natural history of the study group makes interpretation of such data difficult. In approximately half of children diagnosed with epilepsy for which a known cause is identified, the underlying condition is mental retardation or cerebral palsy. ${ }^{10}$ Long-term studies should include evaluations of growth and development. 


\section{CONCLUSIONS}

A wide range of seizure types and epilepsy syndromes present clinically during childhood. The results of pediatric AED development programs have begun to fill in the gaps in our knowledge about efficacy, safety, and appropriate dosing of AEDs. Supported by formulation and toxicology work, the trials designed have been adapted to the special needs of children. In concert with adult trials, trials in refractory seizure types and epilepsy syndromes have the most consistent trial designs and can be expected to continue as part of most pediatric development programs. ${ }^{16,27}$ Adaptations for younger children and infants have included shortening the baseline and treatment phases and counting seizures using video-EEG monitoring. New-onset seizures offer more challenges in trial design from both an ethical and practical perspective.

We still need trials and development programs, particularly for generalized seizures and epilepsy syndromes such as PGTC, JME, and IS. ${ }^{28}$ An evolution of trial design may provide for ethical, practical, and interpretable studies but will not overcome the recruitment challenges due to small numbers of children with various seizure types. Thus, multicenter trials will continue to be designed and conducted on a worldwide basis. ${ }^{26}$

\section{REFERENCES}

1. Pellock JM. Pediatric trials: practical issues. Special populations and trial designs. Adv Neurol 1998;76:167-171.

2. Guidance for Industry: E11 clinical investigation of medicinal products in the pediatric population. U.S. Department of Health and Human Services, Food and Drug Administration, Center for Drug Evaluation and Research, Center for Biologics Evaluation and Research. December 2000. Available at: http://www.fda.gov/ cder/guidance/4099fnl.pdf.

3. Best Pharmaceuticals for Children Act (BPCA). Public Law 107109. January 4, 2002. Available at: http://thomas.loc.gov/bss/d107/ d107laws.html.

4. Pediatric Research Equity Act (PREA). Public Law 108-155. December 3, 2003. Available at: http://thomas.loc.gov/bss/d108/ d108laws.html.

5. Additional safeguards for children in clinical investigations of FDA-regulated products. U.S. Department of Health and Human Services, Food and Drug Administration. April 2001. 66 Federal Register 20589-20600. Available at: http://www.fda.gov/ohrms/ dockets/98fr/042401a.htm.

6. Commission on Antiepileptic Drugs of the International League of Epilepsy. Workshop on antiepileptic drug trials in children. Epilepsia 1991;32:284-285.

7. Sheridan PH, Jacobs MP. The development of antiepileptic drugs for children. Report from the NIH workshop, Bethesda, Maryland, February 17-18, 1994. Epilepsy Res 1996;23:87-92.

8. European Medicines Agency (Paediatric Working Party, Quality Working Party). Reflection paper: formulations of choice for the paediatric population. EMEA/CHMP/PEG/194810/2005. London: European Medicines Agency, 2006. Available at: http://www. emea.europa.eu/pdfs/human/peg/19481005en.pdf.
9. Ouellet D, Bockbrader HN, Wesche DL, Shapiro DY, Garofalo E. Population pharmacokinetics of gabapentin in infants and children. Epilepsy Res 2001;47:229-241.

10. Hauser WA. The prevalence and incidence of convulsive disorders in children. Epilepsia 1994;35(Suppl 2):S1-S6.

11. Camfield PR, Camfield CS, Gordon K, Dooley JM. If a first antiepileptic drug fails to control a child's epilepsy, what are the chances of success with the next drug? J Pediatr 1997;131:821824.

12. Korff C, Nordli DR. Do generalized tonic-clonic seizures in infancy exist? Neurology 2005;65:1750-1753.

13. Wyllie E. The treatment of epilepsy: principles and practice. 4th ed. Philadelphia, PA: Lippincott Williams and Wilkins, 2006.

14. Sullivan JE 3rd, Dlugos DJ. Antiepileptic drug monotherapy: pediatric concerns. Semin Pediatric Neurol 2005;12:88-96.

15. U.S. Department of Health and Human Services Food and Drug Administration. Summaries of medical and clinical pharmacology reviews of pediatric studies [Internet]. Created June 18, 2003; updated periodically. Accessed June 19, 2006. Available at: http:// www.fda.gov/cder/pediatric/Summaryreview.htm.

16. Sahlroot JT, Pledger GW. Reanalysis of existing trial data: what can be learned. Adv Neurol 1998;76:125-131.

17. Pina-Garza JE, Espinoza R, Nordli D, et al. Oxcarbazepine adjunctive therapy in infants and young children with partial seizures. Neurology 2005;65:1370-1375.

18. Nordli DR. Diagnostic difficulty in infants and children. J Child Neurol 2002;17:S28-S35.

19. Motte J, Trevathan E, Arvidsson JFV, et al; Lamictal LennoxGastaut Study Group. Lamotrigine for generalized seizures associated with the Lennox-Gastaut syndrome. N Engl J Med 1997; 337:1807-1812 [Erratum in: N Engl J Med 1998;339:851-852].

20. Sachdeo RC, Glauser TA, Ritter F, Reife R, Lim P, Pledger G. A double-blind, randomized trial of topiramate in Lennox-Gastaut syndrome. Neurology 1999;52:1882-1887.

21. Biton V, Sackellares JC, Vuong A, Hammer AE, Barrett PS, Messenheimer JA. Double-blind, placebo-controlled study of lamotrigine in primary generalized tonic-clonic seizures. Neurology 2005;65:1737-1743.

22. Bourgeois B, Brown L, Pellock JM, et al. Gabapentin (Neurontin (®) monotherapy in children with benign childhood epilepsy with centrotemporal spikes (BECTS): a 36-week, double-blind, placebo-controlled study. Epilepsia 1998;39:163. Abstract.

23. Frank LM, Enlow T, Holmes GL, et al. Lamictal (lamotrigine) monotherapy for typical absence seizures in children. Epilepsia 1999;40:973-979.

24. Trudeau V, Myers S, LaMoreaux L, Anhut H, Garofalo E, Ebersole J. Gabapentin in naïve childhood absence epilepsy: results from two double-blind, placebo-controlled, multicenter studies. J Child Neurol 1996;11:470-475.

25. Mackay MT, Weiss SK, Adams-Webber T, et al. Practice parameter: medical treatment of infantile spasms: report of the American Academy of Neurology and the Child Neurology Society. Neurology 2004;62:1668-1681.

26. Shinnar S, Pellock JM. The trials and tribulations of pediatric drug trials. Neurology 2005;65:1348-1349.

27. French JA, Kanner AM, Bautista J, et al. Efficacy and tolerability of the new antiepileptic drugs. II. Treatment of refractory epilepsy. Report of the Therapeutic and Technology Assessment Subcommittee and Quality Standards Subcommittee of the American Academy of Neurology and the American Epilepsy Society. Neurology 2004;62:1261-1273.

28. French JA, Kanner AM, Bautista J, et al. Efficacy and tolerability of the new antiepileptic drugs. I. Treatment of new onset epilepsy. Report of the Therapeutic and Technology Assessment Subcommittee and Quality Standards Subcommittee of the American Academy of Neurology and the American Epilepsy Society. Neurology 2004;62:1252-1260. 